\title{
A Note on a Generalized Elliptic Integral
}

\author{
George H. Weiss
}

(September 13, 1963)

An expansion of

$$
\Omega_{j}(k)=\int_{0}^{\pi} \frac{d \theta}{\left(1-k^{2} \cos \theta\right)^{j+1 / 2}}
$$

in the neighborhood of $k^{2}=1$ is obtained by a method based on an Abelian theorem.

In a recent paper Epstein and Hubbell have given a short table of the function

$$
\Omega_{j}(k)=\int_{0}^{\pi} \frac{d \theta}{\left(1-k^{2} \cos \theta\right)^{j+1 / 2}}, j=0,1,2, \ldots
$$

which is important in certain problems in radiation physics, [1]. ${ }^{1}$ It is simple to find a power series expansion for $\Omega_{j}(k)$ by expanding the denominator and integrating term by term. It is somewhat more difficult to find an approximation to $\Omega_{j}(k)$ which is valid for $k^{2}$ close to one, and such an approximation is not found in [1] except in the cases $j=0,1$. It is the purpose of this note to furnish such an approximation.

Let us use the identity

$$
\frac{1}{z^{j+1 / 2}}=\frac{1}{\Gamma(j+1 / 2)} \int_{0}^{\infty} t^{j-1 / 2} e^{-z t} d t \quad \operatorname{Re}(z)>0
$$

to write eq (1) in the form

$$
\Omega_{j}(k)=\frac{1}{\Gamma(j+1 / 2)} \int_{0}^{\pi} d \theta \int_{0}^{\infty} t^{j-1 / 2} e^{-\left(1-k^{2} \cos \theta\right) t} d t .
$$

It is not difficult to justify an interchange of orders of integration. The integration over $\theta$ can then be performed by making use of the Bessel function formula

$$
\frac{1}{\pi} \int_{0}^{\pi} e^{k^{2 t} \cos \theta} d \theta=I_{0}\left(k^{2} t\right)
$$

where $I_{0}(x)$ is a modified Bessel function.

Hence $\Omega_{j}(k)$ is

\footnotetext{
' Figures in brackets indicate the literature references at the end of this paper.
}

$\Omega_{j}(k)=\frac{\pi}{\Gamma\left(j-\frac{1}{2}\right)} \int_{0}^{\infty} t^{j-1 / 2} e^{-t} I_{0}\left(k^{2} t\right) d t$

$$
=\frac{\pi}{k^{2 j+1} \Gamma\left(j-\frac{1}{2}\right)} \int_{0}^{\infty} e^{-x\left(\frac{1-k^{2}}{k^{2}}\right)} x^{j-1 / 2} e^{-x} I_{0}(x) d x .
$$

We have therefore exposed $\Omega_{j}(k)$ as a Laplace transform in which the coefficient in the first exponential, $\left(1-k^{2}\right) / k^{2}$, approaches zero as $k^{2}$ approaches 1 . We can now apply an Abelian theorem for Laplace transforms, [2], to determine the behavior of $\Omega_{j}(k)$ in the neighborhood of $k^{2}=1$. To do this we note the asymptotic expansion

$$
e^{-x} I_{0}(x) \sim \frac{1}{\sqrt{2 \pi x}} \sum_{n=0}^{\infty} \frac{(-1)^{n}}{(2 x)^{n}} \frac{\Gamma\left(\frac{1}{2}+n\right)}{\Gamma\left(\frac{1}{2}-n\right)} \frac{1}{n !}
$$

as $x$ tends to infinity. Substituting eq (6) into eq (5) we find for the asymptotic expansion of $\Omega_{j}(k)$ :

$$
\begin{aligned}
& \Omega_{j}(k) \sim \sqrt{\frac{\pi}{2}} \frac{1}{k^{2 j+1} \Gamma\left(j-\frac{1}{2}\right)}\left\{\sum_{n=0}^{j-1} \frac{(-1)^{n} \Gamma\left(\frac{1}{2}+n\right)}{2^{n} \Gamma\left(\frac{1}{2}-n\right)}\right. \\
& \frac{(j-n-1) !}{n !}\left(\frac{k^{2}}{1-k^{2}}\right)^{j-n} \\
& \left.+\sum_{n=j}^{\infty} \frac{(-1)^{j}}{2^{n}} \frac{\Gamma\left(\frac{1}{2}+n\right)}{\Gamma\left(\frac{1}{2}-n\right)} \frac{1}{(n-j) !}\left(\frac{1-k^{2}}{k^{2}}\right)^{n-j} \ln \left(\frac{1-k^{2}}{k^{2}}\right)\right\} .
\end{aligned}
$$

Another useful representation for $\Omega_{j}(k)$ can be obtained by noting that the Legendre function $P_{n-1 / 2}$ $(\cosh \eta)$ can be written, [3],

$$
P_{n-1 / 2}(\cosh \eta)=\frac{1}{\pi} \int_{0}^{\pi} \frac{d \theta}{(\cosh \eta+\sinh \eta \cos \theta)^{n+1 / 2}}
$$


from which it follows that

$$
\begin{aligned}
\Omega_{j}(k)= & \frac{\pi}{\left(1-k^{2}\right)^{\frac{2 j+1}{4}}} P_{j-1 / 2}\left(\frac{1}{\left(1-k^{4}\right)^{1 / 2}}\right) \\
& =\frac{\pi}{\left(1+k^{2}\right) \frac{2 j+1}{2}}{ }_{2} F_{1}\left(\frac{1}{2}, j+\frac{1}{2} ; 1 ; \frac{2 k^{2}}{1+k^{2}}\right) .
\end{aligned}
$$

However, a derivation of asymptotic behavior starting with eq (9) is not as direct as the proof we have given.

\section{References}

[1] L. F. Epstein and J. H. Hubbell, Evaluation of a generalized elliptic-type integral, J. Res. 67B (Math. and Math. Phys.) No. 1,1 (1963).

[2] G. Doetsch, Theorie und Anwendung der Laplace Transformation (Dover Reprint, 1943).

[3] I. M. Ryshik and I. S. Gradstein, Tables of series, products and integrals (VEB Deutscher Verlag der Wissenschaften, Berlin, 1957).

(Paper 68B1-108) 